\title{
Spallation Neutron Source to Provide Facilities to Conduct World-Class Science
}

Neutrons, because they are electrically uncharged, are a unique probe of the structure of matter. Neutron experiments can give precise information about the positions and motion of individual atoms. In addition, neutrons give complementary information to that obtainable from both nuclear magnetic resonance and synchrotron light scattering. Neutron scattering can be used to study residual strain in materials, chemical and biological reactions in "real time," the vortex lattice structure in superconductors, the structure of polymer interfaces and surfaces, and protein dynamics, to name just a few applications.

One of the things which becomes immediately clear upon talking to researchers who use neutron sources is that a dearth of beam lines are available within the United States, and those that are operating are greatly oversubscribed. Some estimates show an oversubscription rate of at least a factor of three, and that is from the scientists and engineers who are still trying to get time at the facilities. Others say that they have given up trying to get time at neutron facilities and will have to do without the results which neutron diffraction and scattering might yield. Currently, most would agree that the United States lags behind the rest of the world in neutron capability. The community of researchers is still relatively small due to the lack of available beam lines. U.S. scientists who need a high flux of neutrons must travel abroad to perform their experiments.

The distribution of experimenters by scientific discipline differs from that in Europe. Most U.S. neutron users are either physical scientists or materials scientists and engineers, with a very small but growing proportion of biologists. Another issue facing the neutron community is how to train the next generation of scientists and engineers without the very best instru- ments and adequate number of beam lines.

Many of these problems and bottlenecks will be addressed when work begins on the planned Spallation Neutron Source (SNS) about six years from now. The principal reason for implementing the SNS is to enable a high level of science and engineering. The greater beam intensity at the SNS will mean that more experiments can be performed in less time. In addition, some experiments, previously impossible because the beam flux of neutrons was inadequate, will now be feasible. Also a spallation source where the instruments are optimized to take into account the beam characteristics will provide a complement to the types of measurements which can be made at a reactor source. The construction of the SNS provides a golden opportunity to design a suite of instruments that will enable world-class science.

In the Fiscal Year 1999 budget, the Senate and Congress approved spending \$130 million in the Department of Energy's appropriation bill to begin construction of the SNS at Oak Ridge National'Laboratory. The initial project, to be completed in 2005, is expected to cost $\$ 1.3$ billion and will provide the scientific and engineering community with a facility that will be one of the best in the world. Once completed, the SNS will be the last type of major facility recommended by the Seitz-Eastman study of the National Research Council in 1984.

The SNS is a collaborative project involving five national laboratories. Lawrence Berkeley National Laboratory will design the ion source. The linear accelerator (linac) will be designed by Los Alamos National Laboratory. Brookhaven National Laboratory will design the accumulator ring. Oak Ridge National Laboratory will design the target, and both Oak Ridge and Argonne National Laboratories will design the experiment systems. A suite of 10 instruments will be included in the initial construction. Design concepts are being solicited from the neutron user community. An additional eight instruments could be added at a later time.

One of the features of the project is that the facility is designed to be upgradeable. The initial plans call for a facility operating at $1 \mathrm{MW}$ of beam power with a pulse repetition rate of $60 \mathrm{~Hz}$ and one target hall. At a later date, the ion source operating current could be increased by a factor of two, and with some additional effort, by a factor of four. Furthermore, a second target hall could be added to take advantage of the science available using a lower repetition rate. This upgrade would provide the research community with an additional suite of 18 instruments.

The state of Tennessee has committed $\$ 8$ million for the construction of a Joint Institute for Neutron Science. This will provide visiting scientists and engineers with meeting rooms, offices, laboratories, a communication center, and housing. The building will be housed between the SNS site and the High Flux Ion Reactor currently operational at Oak Ridge. This facility will provide researchers with the amenities currently available at the best synchrotron sources such as the Advanced Photon Source at Argonne National Laboratory.

Once completed, the SNS will provide the U.S. research community with worldclass capabilities. And because the SNS is being designed to be upgradeable, it should serve the research community for many years.

THOMAS A. WEBER

Thomas A. Weber is director of the Division of Materials Research of the National Science Foundation. The views expressed here are those of the author, and not necessarily those of the National Science Foundation.

\section{MATERIALS RESEARCH SOCIETY PUBLIC AFFAIRS WEBSITE http://www.mrs.org/pa/policy/}

- FYI-by Audrey Leath and Richard Jones, American Institute of Physics: Summarizes science policy developments in Washington affecting the science and technology community.

- Centre for Policy Research on Science and Technology (Canada): Conducts focused research on the relationship between public policy, the management of technology, and innovation.

- Parliamentary Office of Science and Technology (U.K.): Provides balanced and objective analysis of science- and technology-based issues.

Technology and Innovation Policy Information Map: Lists science, technology, and innovation policy resources on the Internet. 